\title{
Prediction of Mortality in Chronic Liver Disease Using Non-hepatic Comorbidities: No SI of Relief as Yet
}

\author{
David E. Kaplan ${ }^{1,2}$
}

Published online: 16 November 2016

(c) The Author(s) 2016. This article is published with open access at Springerlink.com

A significant proportion of mortality in patients with chronic hepatitis $\mathrm{C}$ with or without cirrhosis is related to non-hepatic comorbidities such as cardiovascular or psychiatric disease, drug or alcohol use, renal failure, and nonhepatic cancers [1]. Curing underlying hepatitis $\mathrm{C}$ reduces but does not eliminate the higher rates of liver-related and all-cause mortality typical of patients with chronic $\mathrm{HCV}$ infection $[2,3]$. Although $\sim 70 \%$ of the observed mortality reported in large cohorts of patients with chronic hepatitis C-related cirrhosis can be predicted using liver-specific risk prediction models such as Child-Turcotte-Pugh (CTP) or Model for End-Stage Liver Disease (MELD) [4], the remaining variance largely relates to 'unpredictable' (e.g., accidents, homicide, suicide) and more 'predictable' nonhepatic morbidities (e.g., heart disease, chronic lung disease, renal failure, stroke). Optimized systems to predict the remaining variance could have important implications for public policy, and if calculable at the point-of-care, possibly impact shared clinician-patient medical decisionmaking.

Previous attempts to predict non-hepatic mortality in cohorts of patients with liver disease generally have relied on coding-dependent identification of comorbid illness from administrative sources $[1,5,6]$. Both the CharlsonDeyo Index [5] and Cirrhosis Comorbidity Score [1] identify past medical histories of relevant diseases,

David E. Kaplan

dakaplan@mail.med.upenn.edu

1 Division of Gastroenterology, Perelman University of Pennsylvania School of Medicine, 914 BRB, 421 Curie Blvd., Philadelphia, PA 19104, USA

2 Philadelphia VAMC, Research A402A, 3900 Woodland Avenue, Philadelphia, PA 19104, USA including but not limited to coronary artery disease, kidney disease, substance abuse disorders, and cancer, using International Classification of Disease codes from inpatient and/or outpatient claims data, sources not well suited for point-of-care decision-making. Furthermore, the magnitude of improved prediction of all-cause mortality when these risk models are added to liver-specific prediction models appears to be relatively modest [6].

The Schonberg Index (SI), developed to predict 5-year mortality in ambulatory populations age $>65$ years with $\sim 20 \%$ observed 5-year mortality [7], was subsequently shown to have strong concordance with 9-year mortality [9]. The SI includes 11 specific items such as age (per 5 year increments, 65-85), gender, BMI $(>25$ or $\leq 25)$, prior diagnosis of any cancer, presence or absence of diabetes, smoking history, presence or absence of lung disease, the number of hospitalizations in the past year, lack of independence with performance of activities of daily living (e.g., light housework, preparing meals, shopping, taking medications, using the telephone, arranging for personal travel, and managing personal finances), difficulty walking $1 / 4$ mile, and self-reported general health. It can be administered via questionnaire with minimal clinical supervision and is easily scored in real time. Higher scores in the 0-17 range of the index are associated with a higher mortality. For example, a 75-year-old male in fair health, with a history of diabetes, with at least one IADL dependency, and difficulty walking $1 / 4$ mile would have a risk score of 15 , predicting a $42 \%$ chance of mortality in 5 years and a $75 \%$ chance of mortality in 9 years [9]. If evaluation of such a patient in hepatology clinic for consideration of hepatitis $\mathrm{C}$ therapy revealed F0-1 fibrosis based on fibroelastography, could this information be utilized to educate the patient and inform treatment decisions regarding direct-acting antiviral therapy? 
In this issue of Digestive Diseases and Sciences, Natarajan et al. [8] evaluated the accuracy of the SI as a predictor of non-hepatic mortality in a prospective cohort of patients evaluated at a single chronic hepatitis $\mathrm{C}$ center. Over a 5 year period, the investigators recruited more than 1000 treatment-naïve hepatitis C-mono-infected adults during their initial visits to a hepatitis clinic based at a large Veterans Affairs medical center in order to acquire a detailed medical history, sociodemographic risk factors, and laboratory parameters using computer-assisted patient surveys and chart review. In addition to assessing the SI, the investigators also collected self-reported histories regarding cardiovascular disease, alcohol use, and liver staging estimators (Fibrosure ${ }^{\circledR}$ and MELD score) to determine whether or not these parameters could impact the predictive capacity of SI. The investigators then used stepwise Cox proportional hazard models to evaluate the predictive capacity of models including SI for the outcome of all-cause mortality. Due to the nature of the recruitment site, the existing comorbidities and other risk factors for mortality were widely prevalent among the sampled population. The cohort was overwhelmingly male (97.5\%), active tobacco users (60\%), only $25 \%$ underweight, and $93 \%$ had at least some significant alcohol use. Onequarter were diabetic and over half were hypertensive. Approximately $15 \%$ already had been diagnosed with cardiovascular disease; a similar fraction had been diagnosed with chronic obstructive pulmonary disease; and $17 \%$ had been hospitalized at least once in the prior year. Only one-quarter reported that walking 1/4 mile would not pose a difficulty. The mean SI of 8.2 signified a $\sim 13-16 \%$ 5-year mortality risk, a risk estimate independent of the risk attributable to cirrhosis, which was present in one-third of the cohort. In 4.4 years of median follow-up, the observed mortality rate was actually lower, only $9.7 \%$, possibly due to the younger age of the liver disease cohort than the elderly cohort from which the SI was derived. The investigators then determined which variables according to univariate analysis were associated with mortality. Not surprisingly, the presence of cirrhosis was the strongest predictor of mortality, with an overall hazard ratio of 3.3. In multivariable models including cirrhosis, coronary artery disease, and drug use, each unit change of SI was associated with a $20 \%$ increase in mortality.

Strengths of the present study include its sample size, prospective data collection, and comprehensive collection of covariates. The work furthers the evidence base for recognition of the importance of general health and functional status when trying to manage cohorts of patients with liver disease. It also validates that the SI may be a useful tool for clinicians to quantify comorbidities for making liver-related treatment decisions.
The authors indicate that the magnitude of increased risk associated with 1 point changes in SI is similar to the magnitude of increased risk associated with 1 point increases in MELD scores. Nevertheless, unlike MELD, when grouped into quintiles of SI scores, there was a notable nonlinearity of increased risk. SI poorly discriminated differences in mortality risk in non-cirrhotics within the middle three quintiles of risk. Furthermore, among cirrhotics, the SI was poorly discriminative for mortality risks between the 2 nd-3rd and 4 th-5th quintiles. Thus, based on these data, the SI cannot be utilized as a linear variable, but rather must be interpreted as a dichotomized variable associated with high risk of mortality (e.g., $<11$ vs. $\geq 11$ in non-cirrhotics, or $<9$ vs. $\geq 9$ in cirrhotics).

In addition to the nonlinearity of the SI prediction, several other questions need to be addressed before hepatologists should consider widely applying the SI survey. First, is it practical outside of an academic environment to use an 11-variable scale in routine clinical care? The SI can be self-administered to patients using a roughly 2-page questionnaire and is fairly simple to score. Yet, routine administration would incur costs and would require some type of incentive, such as inclusion as a Center for Medicare and Medicaid Service Physician Quality Reporting System quality indicator for which the present data are not sufficiently robust. Second, while standardized, does the SI really provide significantly more information than most clinicians obtain from a routine history and physical? Would clinician behavior change if presented with the SI data during patient care? Such questions need to be addressed prospectively. Third, would patients at high risk of non-hepatic mortality be willing to forgo interventions such as direct-acting antiviral therapy were they presented with SI-based mortality predictions? How would these data impact patient-clinician interactions? Again these questions need to be addressed prospectively.

Application of SI-based risk stratification could theoretically positively impact clinical care. Accurate comorbidity indices could be used by clinicians to avoid application of highly costly or risky screening regimens to patients at high risk of competing mortality. For instance, when applied to a prostate cancer screening population, the SI identified retrospectively that $33 \%$ of the screened population had high risks of mortality and therefore was unlikely to derive clinical benefit from PSA testing [10]. It was theorized, but not tested, that educational interventions could rather have been used by physicians to educate patients to agree to opt-out, rather than undergo, screening. Nonetheless, the SI has never been prospectively tested in such a setting in order to assess its impact on influencing patient choices. Discussing expected mortality with an individual could have unpredictable consequences. Thus, while Natarajan and colleagues should be lauded for taking 
the first steps in the process, much more prospective testing will be needed before the SI can be considered a routine component of clinical evaluations in hepatology clinics.

Open Access This article is distributed under the terms of the Creative Commons Attribution-NonCommercial 4.0 International License (http://creativecommons.org/licenses/by-nc/4.0/), which permits any noncommercial use, distribution, and reproduction in any medium, provided you give appropriate credit to the original author(s) and the source, provide a link to the Creative Commons license, and indicate if changes were made.

\section{References}

1. Jepsen P, Vilstrup H, Lash TL. Development and validation of a comorbidity scoring system for patients with cirrhosis. Gastroenterology 2014;146:147-56; quiz e15-6.

2. Backus LI, Boothroyd DB, Phillips BR, et al. A sustained virologic response reduces risk of all-cause mortality in patients with hepatitis C. Clin Gastroenterol Hepatol. 2011;9:e1.

3. Innes $\mathrm{H}, \mathrm{McD}$ onald $\mathrm{S}$, Hayes $\mathrm{P}$, et al. Mortality in hepatitis $\mathrm{C}$ patients who achieve a sustained viral response compared to the general population. J Hepatol. 2016. doi:10.1016/j.jhep.2016.08. 004.

4. Kaplan DE, Dai F, Skanderson M, et al. Recalibrating the childturcotte-pugh score to improve prediction of transplant-free survival in patients with cirrhosis. Dig Dis Sci. 2016;61: 3309-3320.

5. Myers RP, Hubbard JN, Shaheen AA, et al. Hospital performance reports based on severity adjusted mortality rates in patients with cirrhosis depend on the method of risk adjustment. Ann Hepatol. 2012;11:526-535.

6. Kaplan DE, Dai F, Aytaman A, et al. Development and performance of an algorithm to estimate the child-turcotte-pugh score from a national electronic healthcare database. Clin Gastroenterol Hepatol 2015.

7. Schonberg MA, Davis RB, McCarthy EP, et al. Index to predict 5 -year mortality of community-dwelling adults aged 65 and older using data from the National Health Interview Survey. $J$ Gen Intern Med. 2009;24:1115-1122.

8. Natarajan, Y, White, DL, El-Serag, HB, et al. Role of non-hepatic medical comorbidity and functional limitations in predicting mortality in patients with HCV. Dig Dis Sci. (Epub ahead of print). doi:10.1007/s10620-016-4303-2.

9. Schonberg MA, Davis RB, McCarthy EP, et al. External validation of an index to predict up to 9-year mortality of community-dwelling adults aged 65 and older. J Am Geriatr Soc. 2011;59:1444-1451.

10. Drazer MW, Prasad SM, Huo D, et al. National trends in prostate cancer screening among older American men with limited 9-year life expectancies: evidence of an increased need for shared decision making. Cancer. 2014;120:1491-1498. 